\title{
STUDYROOM: AN APPLICATION FOR STUDENT COLLABORATION
}

\author{
Alexander Emmanuel, Kyle Scharf, and Jarred Szabadi \\ Carleton University \\ aaemman@gmail.com,kylescharf24@gmail.com,jarredszabadi@cmail.carleton.ca
}

\begin{abstract}
The StudyRoom application is an incentivized knowledge-sharing platform to engage student-to-student interaction. It is designed to allow students to discuss course content with each other by posting questions and answers. In addition, students can also award points to helpful content and earn badges for displaying unique study habits or reaching various achievements. Coupled with the question and answer platform, the application also provides tools for professors to assess their student's engagement as well as current academic struggles in a quantifiable manner.
\end{abstract}

Keywords: Gamification, Collaboration, Engagement

\subsection{Background}

Our project members worked through the first two years of university without knowing each other, even though we were in the same program. Our classes were not extremely large, yet we never got to know each other. We officially met on the first day of classes in third year, in a class composed of seven students. We were forced to work together for the course project and our working relationship was formed. We have continued this working relationship to this day and have found that we all understand course topics better when we collaborate as a group. We wanted to give this opportunity to future students, as discussion can be an invaluable asset in the understanding of information.

\subsection{Problem Motivation}

Current learning management systems, such as Moodle, used by academic institutions lack mechanisms to promote student collaboration, and as a result are not fully utilized. Although they usually possess a class forum for discussion, there is no incentive for students to use them. Secondly, a lack of mobile applications to supplement these systems is causing students to use platforms external to the institution's offering. Ultimately, students don't have a viable software application to discuss course topics when they are physically separated from their peers, effectively limiting participation in course content.

Forming study groups can be an invaluable asset to a student's success because it provides an open environment for students to ask questions and find answers. Students need a modern method for group interaction that leverages the benefits of smartphones to provide anytime anywhere access to course discussion. Furthermore, they require a platform tailored towards connecting them to other students in their courses. When students are forced to use other social platforms to collaborate they are limiting their connectivity to a group of friends who may not have the required knowledge to aid them.

Professors also face limitations in the amount of feedback received from students. All discussions that occur on external platforms are effectively hidden, meaning that professors cannot view the academic progress of their students. Professors need a method to connect to their classroom, as this information is useful for gauging their students understanding of course concepts and overall academic progression.

\subsection{Problem Statement}

Current knowledge sharing platforms available to students such as Moodle don't provide an efficient method for group collaboration. As a result, students cannot gain the benefits of collaboration outside of the classroom if they are unable to form a study group. Additionally, professors are limited to receiving student feedback inside the confines of the classroom and during office hours, where only a portion of their student's learning occurs.

\subsection{Our Solution}

Our solution to this problem is an incentive driven knowledge sharing platform which empowers students to achieve greater learning through group collaboration. Our application will also provide students with an avenue to seek discussion and develop friendships with their peers and professors anonymously. StudyRoom leverages techniques from social networking applications and the concept of gamification in order to increase student engagement and provides students with a lightweight and fun knowledge sharing experience.

StudyRoom also provides professors with a powerful analytical tool called Professor Tools. Professor Tools is a set of tools aimed at quantifying the learning experience of students through autonomous statistical analysis of student behavior. Through Professor Tools, professors can gain further 
Proc. 2014 Canadian Engineering Education Association (CEEA14) Conf.

insight into their student's overall academic progression.

\subsection{Gamification}

One major challenge associated with creating a new learning platform was finding a way to encourage students to try the application and then ensuring that they were motivated to keep using it. This prompted the introduction of gamification. The purpose of gamification in the application was to attract and retain students. Once students were consistently using the application, data could be used to create the classroom statistics required for the Professor Tools module.

Student engagement is a key performance indicator that is used to quantify the learning experience. In today's classrooms compulsory techniques such as attendance, PASS sessions, classroom clickers, and submitted in class quizzes are used to drive student engagement. Outside of the classroom, during their optional time, however, there are few discernible methods to get students engaged in course material. The desire to obtain high grades, while being a driving factor to create engagement, is not a method.

Gamification is a strategy to increase user engagement by leveraging concepts from game design, loyalty programs, and behavioural economics. StudyRoom uses three of the core methods of gamification; points, badges, and leaderboards. Meaningful design of these gamified techniques will increase user engagement, align student's goals towards mastery, and drive innovative discussion of course content.

\subsection{Points}

The major principle in the application is user generated content. That is, all content will be generated by the students. More so, all content will also be moderated by the users with the availability to report a post due to inappropriate content. The powerful factor behind user generated content is relevance and quality.

Aligning with the concept of user generated content, all content a user posts will be subjected to a score of quality as rated by the community. Every upvote by another user increases the point total of the post's quality, whereas a downvote decreases the point total to a minimum of 0 . This minimum ensures that users are never discouraged if they post content that may not be extremely helpful to others. Through this approach, students may gain or lose points based on the quality of content they post. Students will have an overall score as a result of all the points they have accumulated across all content they have posted.

\subsection{Badges}

Badges are tokens that represent an achievement of a particular goal. Students will be rewarded with a badge upon completion of a task or passing some sort of threshold within the application. Earning an achievement is compelling to a user because it creates the feeling of success and accomplishment, which brings the student back into the experience. Moreover, badges allow users to track their accomplishments as they attempt to collect them. StudyRoom's implementation of badges is to loosely define how a user can earn them. This way when they do earn a badge they get a surprised feeling that creates a memorable experience.

There are two categories of badges within StudyRoom: quality of content and study habits. Quality of content badges represent quantifiable achievements in a class such as the "Ken Jennings Badge," which is awarded to a student if they have posted a large number of answers that have the highest rating within a discussion. Study habit badges are earned when a student displays a unique study habit such as posting a question every day of the week, answering a question early in the morning and late at night in the same day, or simply participating in all classes the user is enrolled in. Quality of content badges in combination with study habit badges will provide the feeling of accomplishment for all students through the diversity of achievements in the application.

\subsection{Leaderboards}

Perhaps the most intriguing of all gamification techniques is the introduction of a leaderboard aimed at awakening the latent competitive spirit many students possess. Each class will have its own leaderboard where students will be ranked based on their accumulated point value earned from content they posted in that class's discussion. The application will employ a dynamic 5 user leaderboard, in which the current user is always centered and can only view two spots ahead or behind. The purpose is to motivate students to continue to compete for more points than their peers, while not discouraging the less active and lower ranked students. Students will be encouraged to act in a friendly competitive spirit as they attempt to earn points and become the \#1 ranked student in their course.

\subsection{Mobile User Interface}

This platform will have a mobile and web interface to benefit both professors and students. The following are a some of the current applications user interfaces. 


\subsection{Question Feed}

The Question Feed, as shown in Fig. 1, is the main hub of the StudyRoom platform, and is where users will spend the majority of their time while using the application. The feed is populated by the questions posted in each of the classes which the student is currently enrolled in. Filtering options are available to limit the scope of the feed such as specifying the class, time of creation, and total point value.

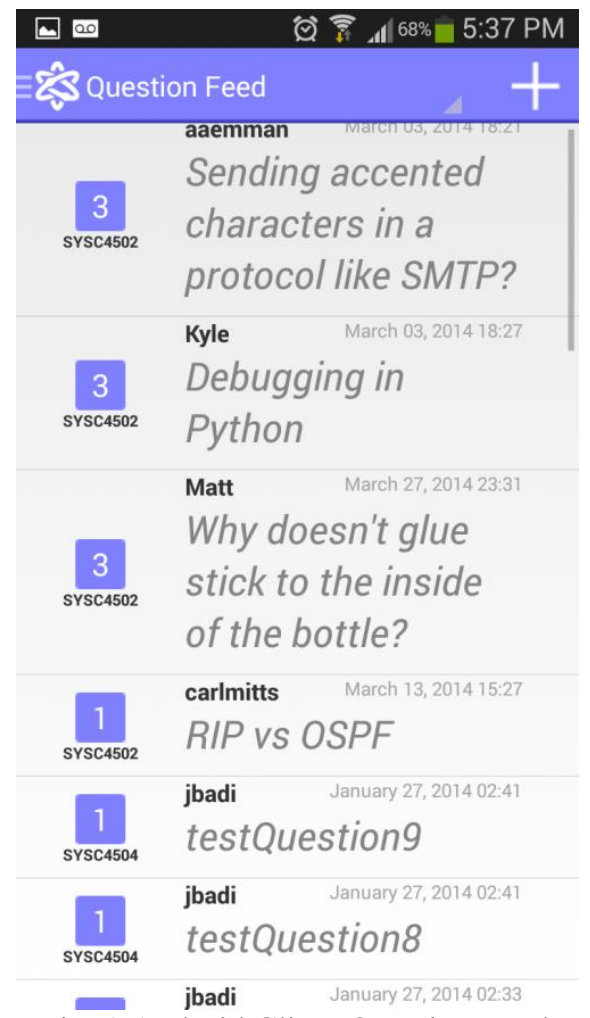

Fig. 1 Android Client Question Feed

\subsection{Answer Page}

The answer page is a detailed view of a question followed by its discussion. Questions will always be displayed at the top of the interface, with lists of comments and answers displayed below if they are available. The answer page is the only interface where users can award points or vote on specific questions and/or answers. The answer page is also the place where users can post answers to questions, post comments on questions and answers, report inappropriate content and if the current user is the author of the featured question, they can mark an answer as being the correct answer to the featured question.

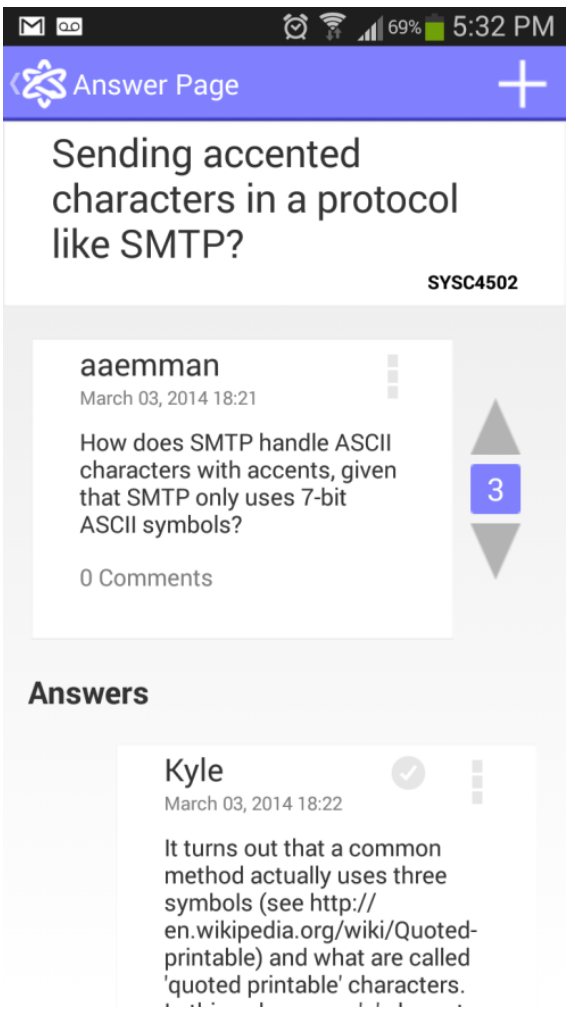

Fig. 2. Android Client Answer Page

\subsection{Web User interface}

Most of the application for the web client will function the same as the mobile client. The major exception is the inclusion of the Professor Tools interface which is unique to the web client.

\subsection{Professor Tools}

A persisting problem in the student-professor relationship is that it is often unidirectional, the professors provide plenty of feedback to the students, however students submit far less feedback to professors. Without this feedback, professors are limited in understanding how the students deal with the content delivered. Figure 3 shows the Professor Tools dashboard, which offers a means to quantify the student learning experience. This quantization is used to make informed observations at a glance.

First, Professor Tools contains a graphical view showing the number of questions posted each month in a given class. This will gauge user participation, but it will also give insight to which classes warrant the most discussion. For example if the professor notices a spike in questions after the date of a certain lecture, that topic can be further explained in the future.

Next, a graphical view showing the most frequently posted topics in the past 7 days. This information shows topics of interest or sources of confusion on a weekly basis, and is an indicator of 
what could be focused on during the lecture time in the future.

It also features a pie chart representing user participation. This view shows the number of students posting questions, answers, questions and answers, and not posting anything. This, for example, could show that a lot of questions are being posted, but few answers, indicating a lack of understanding regarding certain topics. It could also show that there is a high amount of students posting answers indicating a strong understanding on the current content.

Professors will be able to do timeline searches in which they will enter a word and a timeline will be produced showing the volume and frequency of the word as a function of time. This will provide an insight to the topics within the course as well as when they were popular.

Together, these tools will enable a professor to gain knowledge of the learning process taken by students in such a way that will benefit both parties. One important detail is the fact that the data the professor analyzes is detached from the students identity. This system is a rather simple way of providing increased course feedback without subjecting students to any scrutiny or biases along the way.

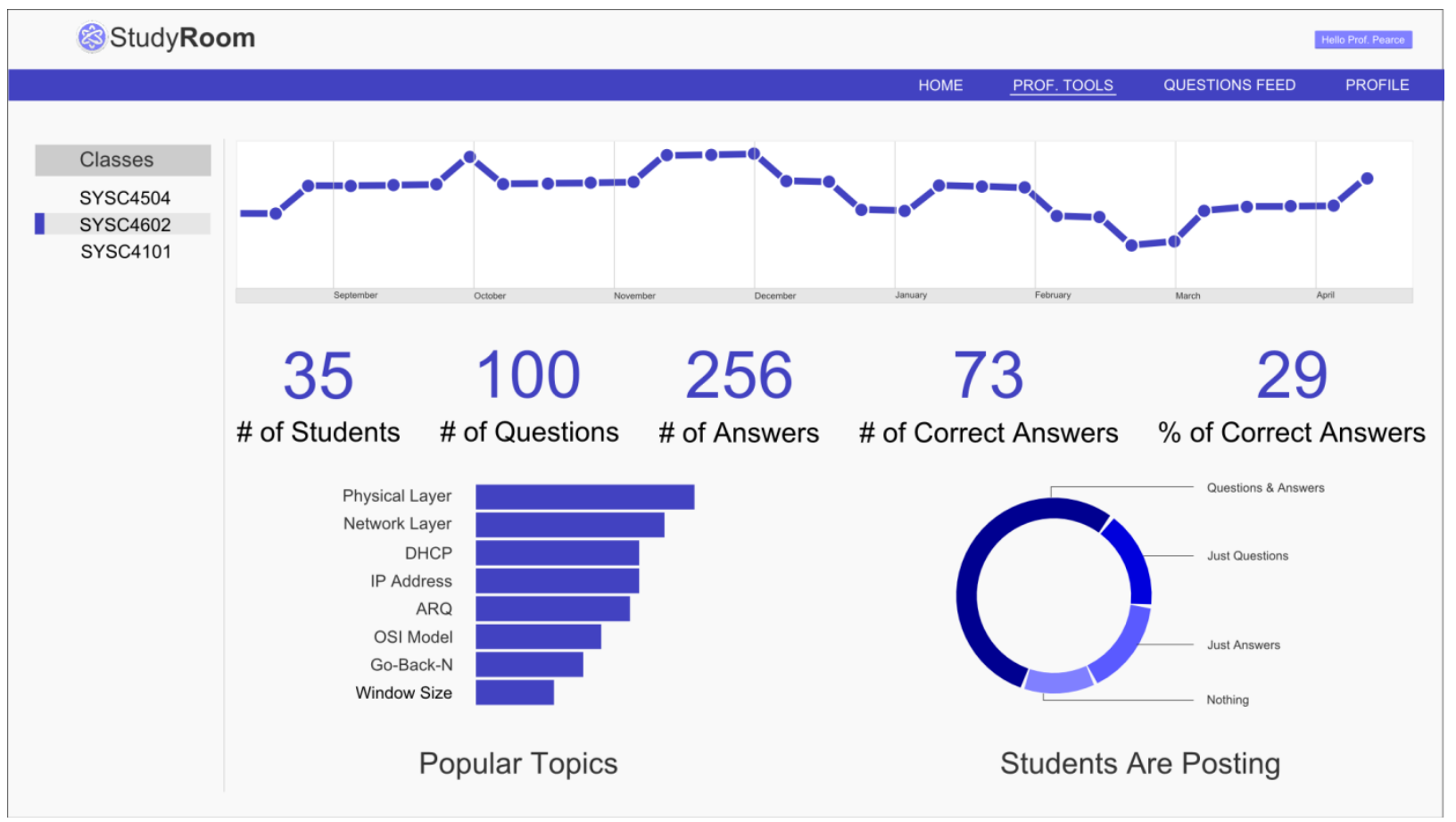

Fig. 3. Web Client Professor Tools

\subsection{Conclusion}

The objective of this project was to create an incentivized knowledge sharing platform to increase student participation outside of the classroom. The goal of the platform was to supplement learning management systems used by academic institutions to enable student-to-student interaction.

\subsection{Acknowledgements}

We would like to thank Professor Trevor Pearce for his guidance and feedback throughout the course of the 2013/2014 academic year at Carleton University. His insight was invaluable to the goals we wished to achieve in completing this project.
We would also like to thank Cheryl Schramm for her assistance over the past 4 years at Carleton University. She was extremely helpful in many of our projects as well as the formation of the StudyRoom team.

\subsection{References}

[1] Gartner Group, "Gartner Says By 2015, More Than 50 Percent of Organizations That Manage Innovation Processes Will Gamify Those Processes", $2011 \mathrm{http} / / /$ www.gartner.com/newsroom/id/1629214 (Last Accessed: January 6, 2014)

[2] M2 Research, "Gamification in 2012: Market Update, Consumer and Enterprise Market Trends", $2012 \mathrm{http}: / / \mathrm{m} 2$ research.com/gamification-2012.htm (Last Accessed: October 25, 2014) 